\title{
On Periodic and Chaotic Regions in the Mandelbrot Set
}

\author{
G. Pastor*, M. Romera, G. Alvarez and F. Montoya \\ Instituto de Fisica Aplicada, Consejo Superior de Investigaciones Cientificas, Serrano 144, \\ 28006 Madrid, Spain
}

\begin{abstract}
We pretend to show here in a graphic and simple way the relation between the periodic and chaotic regions in the Mandelbrot set. Since the relation between the periodic and chaotic regions in a one-dimensional (1D) quadratic map is already well known, we shall base on it to extend the results to the Mandelbrot set. We shall see that in the same way as the period-doubling cascade determines the chaotic bands structure in 1D quadratic maps, the periodic region determines the chaotic region in the Mandelbrot set.
\end{abstract}

PACS: 05.45.+b; 47.20.Ky

Keywords: Mandelbrot set, shrubs, hyperbolic components ordering, Misiurewicz points ordering.

\section{Introduction}

The Mandelbrot set [Mandelbrot, 1980, 1983] can be defined by

$$
M=\left\{c \in \mathbf{C}: f_{c}^{k}(0) \nrightarrow \infty \text { as } k \rightarrow \infty\right\}
$$

where $f_{c}^{k}(0)$ is the $k$-iteration of the complex polynomial function depending on the parameter $c, f_{c}(z)=z^{2}+c, z$ and $c$ complex, for the initial value $z=0$. This set has already been widely studied by many authors [Peitgen \& Richter, 1986; Branner, 1989; Douady \& Hubbard, 1985; Milnor, 1989] because of its historical importance; nevertheless, in a so extraordinary complex set we always have some new approaches to accomplish. From a mathematical point of view the study of this set can be considered almost closed. However, from other points of view there are important shares to be known.

\footnotetext{
*Corresponding author. Phone: +34 915618806; Fax +34 914117651; Email: gerardo@iec.csic.es
} 
Thus, from a practical point of view, in the case of quadratic maps we only can construct a good symbolic dynamics if such quadratic maps are invertible. Since the Mandelbrot set is non-invertible, we do not know to find symbolic sequences [Hao \& Zheng, 1998; Zheng \& Hao, 1990] for their hyperbolic components and Misiurewicz points. Or, yet more simple, if we penetrate in any of the Mandelbrot set filaments we only partially know how their hyperbolic components and Misiurewicz points are ordered.

Nowadays the Mandelbrot set is the more representative paradigm of chaos. According to Devaney [1989], we can give the following definition of a chaotic function $f$ : Let $\mathrm{V}$ be a set. $f$ : $\mathrm{V} \mapsto \mathrm{V}$ is said to be chaotic on $\mathrm{V}$ if: 1) $f$ has sensitive dependence on initial conditions, 2) $f$ is topologically transitive and 3) periodic points are dense in V. Hence, the chaos we are dealing with is not something random since it always fits to a mathematical formula. Therefore, what we refer when we are speaking on chaos is somewhat enormously complicated but deterministic. This chaos is closely related with the order. Thus, we can observe immersed in the chaos periodic components that are part of an ordered structure although in an apparent complex manner.

As we know, three different routes to reach the chaos of a chaotic physical phenomenon have been mentioned: the Myrberg-Feigenbaum route, the intermittence route and the quasiperiodic route [Eckmann, 1981; Lauterborn \& Parlitz, 1988]. We shall treat here he first one, the Myrberg-Feigenbaum route, because we always found this type of route in quadratic maps in both 1D and complex ones, being the Mandelbrot set the more representative of these last ones. This route is completely periodic and with a very strong order that, for the case of 1D quadratic maps, obeys to a very simple law: the well known period-doubling cascade.

In the same way as we use the Mandelbrot set to study the complex case, to study 1D quadratic maps we use the real Mandelbrot map, $x_{n+1}=x_{n}^{2}+c$, which is the intersection of the Mandelbrot set and the real axis, and is defined for the parameter values $-2 \leq c \leq 1 / 4$. In this real Mandelbrot map there are several kinds of points according to the multiplier in the fixed points, $x_{f p}$, of the orbit $\lambda=\left|d\left(f_{c}(x)\right) / d x\right|_{x=x_{f p}}$ [Hale \& Koçak, 1991]. A similar definition of the multiplier can be given for the complex case, as can be seen for example in Branner [1989], where now $c$ is defined for the parameter values of the Mandelbrot set, and not only for the values of the real segment $-2 \leq c \leq 1 / 4$.

In both cases, the real case and the complex case, when $\lambda \neq 1$ we have hyperbolic points. The connected components of the $c$-values set for which $f_{c}^{k}(0)$ converges to a $k$-cycle is a 
periodic hyperbolic component [Branner, 1989]. These periodic hyperbolic components verify $\lambda<1$ which means they are stable (if $\lambda=0$ they are superstable). A hyperbolic component is a cardioid or a disk when we are in the complex case, i. e., a two-dimensional (2D) hyperbolic component, and a segment when we are in the real case, i. e., a 1D hyperbolic component. Therefore, we can speak indistinctly of periodic orbits or hyperbolic components.

There are also points where $\lambda>1$ which means they are unstable. These last points are in addition preperiodic and they have been later called Misiurewicz points [Douady, 1985; Misiurewicz \& Nitecki, 1991; Romera et al., 1996; Pastor et al., 2001]. When $\lambda=1$ we have non-hyperbolic points. These points correspond to tangent bifurcation points (or cusp points, where a cardioid-like component is born) and to pitchfork bifurcation points (or root points, where a disk-like component is born).

In this paper we try to make evident, in the more clear and graphical possible manner, the relation between the periodic region, which is the route to reach the chaos, and the chaotic region of the Mandelbrot set. To this end, we shall see first the already known relation between the periodic and chaotic regions in 1D quadratic maps, by using, as we pointed out before, the real Mandelbrot map $x_{n}=x_{n+1}^{2}+c$. Since this 1D quadratic map can be considered a subset of the Mandelbrot set, we shall try of expanding later to the Mandelbrot set the results obtained from the $1 \mathrm{D}$ quadratic map.

\section{Relation between periodic and chaotic regions in 1D quadratic maps}

The two more representative 1D quadratic maps are the logistic map and the real Mandelbrot map. However, since all the 1D quadratic maps are topologically conjugate [Grossmann \& Thomae, 1977; Milnor \& Thurston, 1988] we can use any 1D quadratic map to study the others. For this purpose we use, as we said before, the real Mandelbrot map $x_{n+1}=x_{n}^{2}+c$ for $x_{0}=0$.

A real map is defined in the real interval of the parameter values where its orbits are defined; hence, it should be depicted on a segment. However, in order to can "see" better the map, traditionally the bifurcation diagram is depicted. In Fig. 1(a) we have depicted the bifurcation diagram of the real Mandelbrot map. We usually use the neighborhood of the real part of the Mandelbrot set in order to "see" such a map (see, for example Pastor et al. [1996]). In Fig. 1(b) we have depicted the neighborhood of the real part of the complex form of such a map. As we can see in both cases, the map is defined for the following parameter values: $-2 \leq c \leq 1 / 4$, as we had already said. 
As can be seen in Fig. 1(a), there are two clearly differentiated regions separated by the Myrberg-Feigenbaum point. Indeed, if starting from the parameter value $c=1 / 4$ we go towards the left by decreasing the parameter value, we observe that the orbit has period 1 between $b_{0}$ and $b_{1}$, period 2 between $b_{1}$ and $b_{2}$, and, in general, period $2^{n}$ between $b_{n}$ and $b_{n+1}$. This periodic region is the very well known period-doubling cascade mentioned before, and finishes in the Myrberg-Feigenbaum point, MF, which is the gate to reach the chaos. When we cover the periodic region from $c=1 / 4$ to MF we follow the only possible route to reach the chaos, and we name this route the periodic route.

The chaotic region expands from the Myrberg-Feigenbaum point to $c=-2$, and, as we pointed out before, we can observe periodic components in this chaotic region. When we cover the chaotic region from MF to $c=-2$ we follow the only possible route to reach the tip, and we name this route the chaotic route. Summarizing, the set of points $c$ of the real Mandelbrot map is the union of two disjoint sets of points corresponding to two clearly differentiated regions: the periodic region which is defined between $\mathrm{MF} \leq c \leq 1 / 4$, and the chaotic region, which is defined between $-2 \leq c \leq \mathrm{MF}$.

As we have just seen, if in the periodic region we start from the parameter value $c=1 / 4$, and we go towards the left in Fig. 1(a), for decreasing values of $c$, we have a period-1 orbit followed by a period- 2 orbit, a period- 4 orbit , a period- 8 orbit, and so on until the MyrbergFeigenbaum point, that is the well known period-doubling cascade of the period-1 orbit. Let us see what is about the chaotic region. To this end, we are going to start from the parameter value $c=-2$ and we are going to go toward the right in Fig. 1(a), in the opposite direction as we did in the periodic region, i. e. now we follow increasing values of the parameter $c$. As we can see in the figure, and as in addition is well known, there is only one chaotic band between $m_{0}$ and $m_{1}$. In $m_{1}$, the chaotic region splits into two chaotic bands. When we reach $m_{2}$, each chaotic band splits into two new ones, giving four chaotic bands in total. This process continues uninterruptedly as far as the Myrberg-Feigenbaum point (the points $m_{0}, m_{1}, m_{2}, \ldots$ are the well known merging points of the chaotic bands) [Lorenz, 1980]. Therefore, we have something very singular though well known: there is also a period-doubling cascade of the chaotic bands. Between $m_{0}$ and $m_{1}$ we have the so-called chaotic band of period $1\left(2^{0}\right), \mathbf{B}_{0}$; between $m_{1}$ and $m_{2}$ the chaotic band of period $2\left(2^{1}\right), \mathbf{B}_{1}$; between $m_{2}$ and $m_{3}$ the chaotic 
band of period $4\left(2^{2}\right), \mathbf{B}_{2} ; \ldots$; between $m_{n}$ and $m_{n+1}$ the chaotic band of period $2^{n}, \mathbf{B}_{n}$; and so on until the Myrberg-Feigenbaum point.

Hence we have found that the periodic and chaotic regions are closely related despite their opposite nature. At first sight, we think that the periodic orbits (or hyperbolic components) of the periodic region which appear when, starting from $c=1 / 4$, we go towards the left: $1,2,4,8, \ldots$ (i. e., $2^{0}, 2^{1}, 2^{2}, 2^{3}, \ldots$ ) should be related with the chaotic bands of the chaotic region which appear when, starting from $c=-2$, we go towards the right: $\mathbf{B}_{0}, \mathbf{B}_{1}$, $\mathbf{B}_{2}, \mathbf{B}_{3}, \ldots$. That is to say, the periodic orbit of period $2^{0}$, placed the first one on the right end of Fig. 1(a), should be related with the chaotic band of period $2^{0}, \mathbf{B}_{0}$, placed the first one on the left end of Fig. 1(a); and in general, the periodic orbit of period $2^{n}$, placed the $(n+1)$ th one on the right end of Fig. 1(a), should be related with the chaotic band of period $2^{n}, \mathbf{B}_{n}$, placed the $(n+1)$ th one on the left end of Fig. 1(a). This relation has clearly been made evident in our work on structural components [19]. Indeed, as we can see there, if we name $\mathbf{G}_{0}$ ( $\mathbf{G}$ of gene) to the pattern or symbolic sequence of the orbit (or hyperbolic component) of period $2^{0}$, the successive harmonics (there named F-harmonics) of $\mathbf{G}_{0}$ give the symbolic sequences of the last appearance hyperbolic components of $\mathbf{B}_{0}$. That is to say, if we start from the gene $\mathbf{G}_{0}$ and we calculate its successive harmonics: $H^{(m)}\left(\mathbf{G}_{0}\right), 2 \leq m \leq \infty$, we obtain the last appearance hyperbolic components, or structural components, of the chaotic band $\mathbf{B}_{0}$ [Pastor et al., 1997]. Again, we have the same for $\mathbf{G}_{1}$ and $\mathbf{B}_{1}$ where the hyperbolic component of period $2^{1}$, that we name gene $\mathbf{G}_{1}$, generates the last appearance hyperbolic components, or structural components, of the chaotic band of period $2^{1}, \mathbf{B}_{1}$. And so on, giving in the general case that the chaotic band $\mathbf{B}_{n}$ is generated starting from the gene $\mathbf{G}_{n}$ for any value $0 \leq n \leq \infty$. These calculations, that had been accomplished using symbolic sequences in Pastor et al. [1997], have been generalized in Pastor et al. [2002] by using external arguments.

Characteristic Misiurewicz points are excellent indicators in order to find the gene of a chaotic band. As we know from Romera et al. [1996] and Pastor et al. [2001], the more prominent and visible Misiurewicz points of a chaotic band are the characteristic Misiurewicz points of such a chaotic band. In a specific chaotic band, these characteristic Misiurewicz points have the same period, and it coincides with the period of the chaotic band and with the 
period of the gene of such a chaotic band. Therefore, by knowing the period of the characteristic Misiurewicz points we know the period of the gene. Thus, in the period- $1\left(2^{0}\right)$ chaotic band $\mathbf{B}_{0}$, the characteristic Misiurewicz points are $M_{n^{*}, 1}$, then the gene of $\mathbf{B}_{0}$ has period 1 ; in the period-2 $\left(2^{1}\right)$ chaotic band $\mathbf{B}_{1}$, the characteristic Misiurewicz points are $M_{n^{*}, 2}$, then the gene of $\mathbf{B}_{1}$ has period $2 ; \ldots$ and in the period- $2^{n}$ chaotic band $\mathbf{B}_{n}$, the characteristic Misiurewicz points are $M_{n^{*}, 2^{n}}$, then the gene of $\mathbf{B}_{n}$ has period $2^{n}$. In any chaotic band we can find non-characteristic Misiurewicz points with greater periods than the period of the chaotic band but never with smaller periods.

In Fig. 2 we show the sketch of the real Mandelbrot map by using, as in Fig. 1(b), the neighborhood of the real part of the Mandelbrot set (let us remember that the real Mandelbrot map is the intersection of this figure and the real axis, i. e. a segment; that is why we use this two-dimensional figure in order to better "see" such a map). The periodic and chaotic regions, which are separated by the Myrberg-Feigenbaum point, are clearly differentiated in this Fig. 2. We also make evident in this figure the relation between the hyperbolic components of the period-doubling cascade $\mathbf{G}_{n}, 0 \leq n \leq \infty$, and the corresponding chaotic bands $\mathbf{B}_{n}, 0 \leq n \leq \infty$. The Myrberg-Feigenbaum point is the boundary between both regions, not a boundary that separates but a boundary that unites. Let us note that each region seems the specular image of the other but with a "diabolic mirror", the point MF, which transforms what is periodic in chaotic, and vice versa. It seems as if when we cross the period-doubling cascade towards the chaos we were determining how the chaos will be, as if each hyperbolic component of the period-doubling cascade were the index which shows the developing of the corresponding chaotic band.

Before finishing with the real Mandelbrot map, let us take into account some additional considerations on its periodic and chaotic regions. Thus, the periodic region is a connected set constituted by a segment beginning at the cusp, which is a tangent bifurcation placed in $c=1 / 4$, and ending in MF. We also can admit the chaotic region to be connected. Otherwise, as we saw in the introduction, the real Mandelbrot map has different types of points according to the value of the multiplier $\lambda$. In the chaotic region we can find the whole type of points that we saw there. However, in the periodic region we can only find points where multiplier is $\lambda \leq 1$. When $\lambda=1$ we have either the unique tangent bifurcation of the periodic region (the cusp or the birth of the period-1 periodic orbit) or any of the infinity of pitchfork bifurcations that we have when we cross from a periodic orbit to the following one in the period-doubling 
cascade. When $\lambda \leq 1$ we have stable periodic points (superstable ones if $\lambda=0$ ). However, in this periodic region there is no point with $\lambda>1$, i. e., there is no Misiurewicz point.

If we had used the Lyapunov exponent of any point of the real Mandelbrot map instead of the multiplier of these points, in a similar way as Shaw [1981] did in the case of the logistic map, we would have a parallel result. In this case, in the chaotic region the Lyapunov exponent has all the possible values: positive, negative and null. Nevertheless, in the periodic region the Lyapunov exponent only can have either null values (corresponding to the two types of bifurcations) or negative values (corresponding to stable and superstable points), but there is no positive value which correspond to Misiurewicz points, which are inexistent in this region.

We have seen the relation between the periodic and chaotic regions in the real Mandelbrot map, what was already very familiar to us. Let us see now the relation between the periodic and chaotic regions in the Mandelbrot set, what is much less familiar to us, and to a certain extent unknown.

\section{Relation between periodic and chaotic regions in the Mandelbrot set}

As we did for the real Mandelbrot map, we shall begin by splitting the Mandelbrot set into a periodic region and a chaotic region. Since the two-dimensional case is not as simple as the one-dimensional case, we shall treat separately the study of both regions by our convenience.

\subsection{Periodic region of the Mandelbrot set}

In accordance with other authors, for example Devaney [1995], we name primary hyperbolic components of the Mandelbrot set (what he names primary bulbs) those directly attached to the main cardioid. We can associate a rational number $\frac{q}{p}$, the rotation number, to each primary hyperbolic component, and we can use this rotation number to identify each primary hyperbolic component [Devaney, 1995]. This primary hyperbolic component $\frac{q}{p}$, that we normally write as $\frac{q_{1}}{p_{1}}$, is related to the angle $\alpha$ which varies from 0 to $2 \pi$ when, starting from the cusp, it turns counter clockwise to come back to the cusp (see Fig. 3 where a sketch with some hyperbolic components of the upper part of the Mandelbrot set periodic region is shown). 
We denominate secondary hyperbolic component $\frac{q_{1}}{p_{1}} \cdot \frac{q_{2}}{p_{2}}$ to the hyperbolic component attached to the primary hyperbolic component $\frac{q_{1}}{p_{1}}$, in the relative position that $\frac{q_{2}}{p_{2}}$ should have with regard to the main cardioid [Romera et al., 2003]. By generalising, we denominate $N$-ary hyperbolic component $\frac{q_{1}}{p_{1}} \cdot \frac{q_{2}}{p_{2}} \cdots \frac{q_{N}}{p_{N}}$ the hyperbolic component attached to the $(N-1)$-ary hyperbolic component $\frac{q_{1}}{p_{1}} \cdot \frac{q_{2}}{p_{2}} \cdots \frac{q_{N-1}}{p_{N-1}}$, in the relative position that $\frac{q_{N}}{p_{N}}$ should have with regard to the main cardioid.

We introduce now the generation route of a $N$-ary hyperbolic component $\frac{q_{1}}{p_{1}} \cdot \frac{q_{2}}{p_{2}} \cdots \frac{q_{N}}{p_{N}}$, that we represent as $\operatorname{gr}\left(\frac{q_{1}}{p_{1}} \cdot \frac{q_{2}}{p_{2}} \cdots \frac{q_{N}}{p_{N}}\right)$, because we shall use it afterwards. We denominate generation route of the $N$-ary hyperbolic component $\frac{q_{1}}{p_{1}} \cdot \frac{q_{2}}{p_{2}} \cdots \frac{q_{N}}{p_{N}}$ to the ordered set of hyperbolic components that we find in the route to reach $\frac{q_{1}}{p_{1}} \cdot \frac{q_{2}}{p_{2}} \ldots \frac{q_{N}}{p_{N}}$ starting from the period-1 main cardioid $\frac{1}{1}$. That is to say, the generation route is the ordered set of $N$ hyperbolic components: $\frac{1}{1}, \frac{q_{1}}{p_{1}}, \frac{q_{1}}{p_{1}} \cdot \frac{q_{2}}{p_{2}}, \frac{q_{1}}{p_{1}} \cdot \frac{q_{2}}{p_{2}} \cdot \frac{q_{3}}{p_{3}} \ldots \frac{q_{1}}{p_{1}} \cdot \frac{q_{2}}{p_{2}} \ldots \frac{q_{N-2}}{p_{N-2}}$ and $\frac{q_{1}}{p_{1}} \cdot \frac{q_{2}}{p_{2}} \ldots \frac{q_{N-1}}{p_{N-1}}$.

We have normally named the main cardioid with its symbolic sequence C. However, if we want to name it by means of a rational number, we have to name it $\frac{1}{1}$ since the period is 1 (then denominator 1) and it is the first -and the unique- with period 1 (then numerator 1). In some generalizations it would be better to write a $\mathrm{N}$-ary hyperbolic component as $\frac{q_{0}}{p_{0}} \cdot \frac{q_{1}}{p_{1}} \cdot \frac{q_{2}}{p_{2}} \cdots \frac{q_{N}}{p_{N}}$ where always $\frac{q_{0}}{p_{0}}=\frac{1}{1}$, i.e. $\frac{1}{1} \cdot \frac{q_{1}}{p_{1}} \cdot \frac{q_{2}}{p_{2}} \cdots \frac{q_{N}}{p_{N}}$. However, by our convenience, we normally use here the notation without $\frac{1}{1}$.

The set of hyperbolic components of the Mandelbrot set periodic region, $\mathrm{P}_{h c}$, is made up of the main cardioid (that can be thought of as the only 0 -ary hyperbolic component) and all the primary, secondary, ..., $N$-ary hyperbolic components. If we denominate any primary hyperbolic component as $h c^{(1)}$, any secondary hyperbolic component as $h c^{(2)}$, and so on, we shall have:

$$
\mathrm{P}_{h c}=\left\{\frac{1}{1}\right\} \cup\left\{h c^{(1)}\right\} \cup\left\{h c^{(2)}\right\} \cup\left\{h c^{(3)}\right\} \cup \cdots
$$

This set, whose sketch of some of its hyperbolic components is shown in Fig. 3, is much more complicated than the simple period-doubling cascade that we had in the onedimensional case. To begin with, let us see its construction. We begin by the main cardioid $\frac{1}{1}$ 
which is the only 0 -ary hyperbolic component. The primary hyperbolic components have the generic form $\frac{q_{1}}{p_{1}}$ (that sometimes we write $\frac{1}{1} \cdot \frac{q_{1}}{p_{1}}$ ), but any $q_{1}$ and $p_{1}$ are not valid. Hyperbolic components can easily be found if, by starting from $0 / 1$ and $1 / 1$, the successive Farey additions are accomplished, as it is shown for example in Devaney [1995]. The secondary hyperbolic components, $\frac{q_{1}}{p_{1}} \cdot \frac{q_{2}}{p_{2}}$, are constructed by placing them attached to the primary hyperbolic component $\frac{q_{1}}{p_{1}}$, in the relative position that $\frac{q_{2}}{p_{2}}$ should have with regard to the main cardioid. And so on.

The set constructed in such a manner is simply connected as in the one-dimensional case. This set begins in the same point as the one-dimensional case, $c=1 / 4+0 i$, the cusp of the main cardioid that is again a tangent bifurcation. As in the one-dimensional case, the main cardioid is the only cardioid of the set, since all the other hyperbolic components of the set are discs (let us remember that in the 1D case this is only a way of speaking, since in this case we only have segments). However, this set has not one unique point to reach the chaos, the Myrberg-Feigenbaum point, as it had the one-dimensional case, but an infinity.

We name now $\mathrm{MF}^{(0)}$ to the Myrberg-Feigenbaum point of the real axis, that in the onedimensional case was named simply MF. By following the period-doubling cascade of any of the primary hyperbolic components we reach what we name primary Myrberg-Feigenbaum points, $\mathrm{MF}^{(1)}$, which are the points where the periodic region ends and the chaotic region begins. In Fig. 3 we can see some of them: $\operatorname{MF}^{(1)}\left(\frac{2}{5}\right), \operatorname{MF}^{(1)}\left(\frac{1}{3}\right), \operatorname{MF}^{(1)}\left(\frac{1}{4}\right)$, etc. Likewise, by following the period-doubling cascade of any of the secondary hyperbolic components we reach what we name secondary Myrberg-Feigenbaum points, $\mathrm{MF}^{(2)}$. In Fig. 3 we can also see some of them: $\operatorname{MF}^{(2)}\left(\frac{1}{3} \cdot \frac{2}{3}\right), \operatorname{MF}^{(2)}\left(\frac{1}{3} \cdot \frac{1}{3}\right), \operatorname{MF}^{(2)}\left(\frac{1}{3} \cdot \frac{1}{4}\right)$ and $\operatorname{MF}^{(2)}\left(\frac{1}{4} \cdot \frac{1}{3}\right)$. Likewise, by following the period-doubling cascade of any of the $N$-ary hyperbolic components we reach what we name $N$-ary Myrberg-Feigenbaum points, $\mathrm{MF}^{(N)}$. In Fig. 3 we can see two of them with $N>2: \mathrm{MF}^{(3)}\left(\frac{1}{3} \cdot \frac{1}{3} \cdot \frac{2}{3}\right)$ for $N=3$ and $\mathrm{MF}^{(4)}\left(\frac{1}{3} \cdot \frac{1}{3} \cdot \frac{2}{3} \cdot \frac{2}{3}\right)$ for $N=4$. Therefore, if we admit, as we did in the introduction $[10,11]$, that the route to reach the chaos crosses the period-doubling cascade of the different types of hyperbolic components, then the boundary of the periodic region is, in addition to the cusp and $\mathrm{MF}^{(0)}$, the infinity of $\mathrm{MF}^{(1)}$, the infinity of $\mathrm{MF}^{(2)}, \ldots$, the infinity of $\mathrm{MF}^{(N)}, \ldots$. We name Myrberg-Feigenbaum route of the $N$-ary hyperbolic component $\frac{q_{1}}{p_{1}} \cdot \frac{q_{2}}{p_{2}} \cdots \frac{q_{N}}{p_{N}}, \operatorname{MFr}\left(\frac{q_{1}}{p_{1}} \cdot \frac{q_{2}}{p_{2}} \cdots \frac{q_{N}}{p_{N}}\right)$, to the period-doubling cascade of 
$\frac{q_{1}}{p_{1}} \cdot \frac{q_{2}}{p_{2}} \cdots \frac{q_{N}}{p_{N}}$. That is to say, the Myrberg-Feigenbaum route of $\frac{q_{1}}{p_{1}} \cdot \frac{q_{2}}{p_{2}} \cdots \frac{q_{N}}{p_{N}}$ is the ordered set of $\infty$ hyperbolic components: $\frac{q_{1}}{p_{1}} \cdot \frac{q_{2}}{p_{2}} \cdots \frac{q_{N}}{p_{N}}, \frac{q_{1}}{p_{1}} \cdot \frac{q_{2}}{p_{2}} \cdots \frac{q_{N}}{p_{N}} \cdot \frac{1}{2}, \frac{q_{1}}{p_{1}} \cdot \frac{q_{2}}{p_{2}} \cdots \frac{q_{N}}{p_{N}} \cdot \frac{1}{2} \cdot \frac{1}{2}, \ldots$

In the one-dimensional case, there is one only possible route to reach the chaos when we cover the periodic region from $c=1 / 4$ to $\mathrm{MF}$, the periodic route. In the periodic region of the Mandelbrot set, since there are an infinity of MF points, we have an infinity of routes to reach the chaos, i.e. we have an infinity of periodic routes. Each one of these periodic routes, $\operatorname{pr}\left(\frac{q_{1}}{p_{1}} \cdot \frac{q_{2}}{p_{2}} \cdots \frac{q_{N}}{p_{N}}\right)$, has two parts. The first part is the generation route that covers from the main cardioid $\frac{1}{1}$ to the $(N-1)$-ary hyperbolic component $\frac{q_{1}}{p_{1}} \cdot \frac{q_{2}}{p_{2}} \cdots \frac{q_{N-1}}{p_{N-1}}$. And the second part, the Myrbeg-Feigenbaum route, is the period-doubling cascade of the $N$-ary hyperbolic component $\frac{q_{1}}{p_{1}} \cdot \frac{q_{2}}{p_{2}} \cdots \frac{q_{N}}{p_{N}}$. Therefore, $\operatorname{pr}\left(\frac{q_{1}}{p_{1}} \cdot \frac{q_{2}}{p_{2}} \cdots \frac{q_{N}}{p_{N}}\right)$ is the route to reach the chaos when we cover the periodic region from the main cardioid to a MF point through the $N$-ary hyperbolic component $\frac{q_{1}}{p_{1}} \cdot \frac{q_{2}}{p_{2}} \cdots \frac{q_{N}}{p_{N}}$.

As in the one-dimensional case, all the points of this set are characterized by having a multiplier $\lambda \leq 1$ and a Lyapunov exponent $\Lambda \leq 0$. When $\lambda=1$ (or $\Lambda=0$ ), we have either a tangent bifurcation, the cusp, or one of the infinity of pitchfork bifurcations or M-furcations (root points). When $\lambda<1$ (or $\Lambda<0$ ), we have a periodic point (superstable if $\lambda=0$ ). But in no case $\lambda>1$ or $\Lambda>0$ in this set, i.e., in this set there is no Misiurewicz point, as it happens in the one-dimensional case.

After having seen the former considerations on the periodic region of the Mandelbrot set, let us see now its chaotic region.

\subsection{Chaotic region of the Mandelbrot set.}

The access to the chaos, as we have said before, takes place through the MyrbergFeigenbaum points $\mathrm{MF}^{(N)}$ where $0 \leq N \leq \infty$. For $N=0$ there is one unique point, $\mathrm{MF}^{(0)}$, but for all the other values of $N$ there are an infinity of them.

If we start from the periodic region and we cross any Myrberg-Feigenbaum point we reach the chaotic region, and we use the shrubs [Romera et al., 2003; Pastor et al., 2005] to represent this chaotic region. The hyperbolic components are as if mounted on the shrub branches. The more prominent Misiurewicz points of the Mandelbrot set are either the nodes where the branches of the shrubs come together or the tips of such shrubs. 
In Fig. 4 we can see the sketch of the shrub of the $N$-ary hyperbolic component $\frac{q_{1}}{p_{1}} \cdot \frac{q_{2}}{p_{2}} \cdots \frac{q_{N}}{p_{N}}, \operatorname{shrub}\left(\frac{q_{1}}{p_{1}} \cdot \frac{q_{2}}{p_{2}} \cdots \frac{q_{N}}{p_{N}}\right)$. As we can note in the figure, the shrub is split into two parts: the branch 0 , main branch or $\operatorname{shrub}_{0}\left(\frac{q_{1}}{p_{1}} \cdot \frac{q_{2}}{p_{2}} \cdots \frac{q_{N}}{p_{N}}\right)$, and the shrub remainder, $\operatorname{shrub}_{\mathrm{r}}\left(\frac{q_{1}}{p_{1}} \cdot \frac{q_{2}}{p_{2}} \cdots \frac{q_{N}}{p_{N}}\right)$ [Romera et al., 2003; Pastor et al., 2005]. That is to say:

$$
\operatorname{shrub}\left(\frac{q_{1}}{p_{1}} \cdot \frac{q_{2}}{p_{2}} \cdots \frac{q_{N}}{p_{N}}\right)=\operatorname{shrub}_{0}\left(\frac{q_{1}}{p_{1}} \cdot \frac{q_{2}}{p_{2}} \cdots \frac{q_{N}}{p_{N}}\right) \cup \operatorname{shrub}_{\mathrm{r}}\left(\frac{q_{1}}{p_{1}} \cdot \frac{q_{2}}{p_{2}} \cdots \frac{q_{N}}{p_{N}}\right)
$$

As we saw in Pastor et al. [2005], the $\operatorname{shrub}_{0}\left(\frac{q_{1}}{p_{1}} \cdot \frac{q_{2}}{p_{2}} \cdots \frac{q_{N}}{p_{N}}\right)$ is formed by the infinite number of chaotic bands of the $N$-ary hyperbolic component $\frac{q_{1}}{p_{1}} \cdot \frac{q_{2}}{p_{2}} \cdots \frac{q_{N}}{p_{N}}$, that is to say:

$$
\operatorname{shrub}_{0}\left(\frac{q_{1}}{p_{1}} \cdot \frac{q_{2}}{p_{2}} \ldots \frac{q_{N}}{p_{N}}\right)=\mathbf{B}_{0}\left(\frac{q_{1}}{p_{1}} \cdot \frac{q_{2}}{p_{2}} \cdots \frac{q_{N}}{p_{N}}\right) \cup \mathbf{B}_{1}\left(\frac{q_{1}}{p_{1}} \cdot \frac{q_{2}}{p_{2}} \cdots \frac{q_{N}}{p_{N}}\right) \cup \cdots \cup \mathbf{B}_{\infty}\left(\frac{q_{1}}{p_{1}} \cdot \frac{q_{2}}{p_{2}} \cdots \frac{q_{N}}{p_{N}}\right)
$$

Likewise, as we saw in Romera et al. [2003] and Pastor et al. [2005], the shrub remainder of the $N$-ary hyperbolic component $\frac{q_{1}}{p_{1}} \cdot \frac{q_{2}}{p_{2}} \cdots \frac{q_{N}}{p_{N}}, \operatorname{shrub}_{\mathrm{r}}\left(\frac{q_{1}}{p_{1}} \cdot \frac{q_{2}}{p_{2}} \cdots \frac{q_{N}}{p_{N}}\right)$, is constituted by $N$ subshrubs. Hence:

$$
\operatorname{shrub}_{\mathrm{r}}\left(\frac{q_{1}}{p_{1}} \cdots \frac{q_{N}}{p_{N}}\right)=\operatorname{subshrub}_{1}\left(\frac{q_{1}}{p_{1}} \cdots \frac{q_{N}}{p_{N}}\right) \cup \operatorname{subshrub}_{2}\left(\frac{q_{1}}{p_{1}} \cdots \frac{q_{N}}{p_{N}}\right) \cup \cdots \cup \operatorname{subshrub}_{N}\left(\frac{q_{1}}{p_{1}} \cdots \frac{q_{N}}{p_{N}}\right)
$$

From each one of the structural nodes of the first, second, ..., penultimate and last subshrub $p_{N}, p_{N-1}, \ldots, p_{2}$ and $p_{1}$ structural branches emanate. In general, the number of structural branches emanating from a structural node of the $\operatorname{subshrub}_{\mathrm{i}}\left(\frac{q_{1}}{p_{1}} \cdot \frac{q_{2}}{p_{2}} \cdots \frac{q_{N}}{p_{N}}\right)$ is $p_{N-i+1}$ [Romera et al., 2003]. The first subshrub rises in the main node which is the end of the main branch, branch 0 or $\operatorname{shrub}_{0}\left(\frac{q_{1}}{p_{1}} \cdot \frac{q_{2}}{p_{2}} \cdots \frac{q_{N}}{p_{N}}\right)$. However, each one of the following subshrubs has an infinity of portions each one of them rising from a premier tip [Romera et al., 2003] of the previous subshrub.

As we have seen in Romera et al. [2003], the figure of a shrub is enormously complicated. Starting from each branch representative of the first subshrub we can reach an infinity of tips, one for each unlocked path we can follow. But, from each one of these branch representatives we can follow one unique premier unlocked path, and reach one unique premier tip, which is not a crisis point as the former tips but the beginning of a portion of the 
second subshrub [Romera et al., 2003]. The set of the infinite number of shrub portions with their infinity of ramifications constitutes the second subshrub. This is repeated for the following subshrubs: from the infinite number of premier tips of each subshrub rise the shrub portions that constitute the following subshrub, until reaching the last one.

In each one of the primary, secondary, $\ldots, N$-ary, $\ldots$ shrubs there are all the possible types of points according to their multiplier $\lambda$ that can be greater, equal or less than 1 . When $\lambda>1$ we have an unstable point (Misiurewicz point). When $\lambda=1$ we have either a tangent bifurcation (the cusp, the bird of a cardioid) or a pitchfork bifurcation (or root point which is either the touch point between two hyperbolic components of the period-doubling cascade or a M-furcation which is the touch between a $N$-ary hyperbolic component and a $(N+1)$-ary one, not belonging to the period-doubling cascade). And finally, when $\lambda<1$ we have the stable points (superstable ones if $\lambda=0$ ). We can do a similar analysis for the Lyapunov exponent, which also can have in this chaotic region anyone of the possible values, positive values for the Misiurewicz points, negative values for the stable and superstable points, and null values for tangent and pitchfork bifurcations or M-furcations. Therefore, we have the same situation that we had in the chaotic region of the one-dimensional case.

We can also admit a shrub to be connected. Then, the first subshrub is also connected but the second, ..., Nth subshrubs are not connected since each one of them are separated into an infinity of portions.

\subsection{Relation between periodic and chaotic regions in the Mandelbrot set.}

As we have just seen in the two previous sections, the periodic and chaotic regions of the Mandelbrot set not only have a different nature but, in addition, at first sight their shape are so different that it is difficult to think they can be related. However, they are related a lot as we shall see next. In Fig. 5 we show the sketch of a $N$-ary hyperbolic component shrub, the $\operatorname{shrub}\left(\frac{q_{1}}{p_{1}} \cdot \frac{q_{2}}{p_{2}} \cdots \frac{q_{N}}{p_{N}}\right)$, that we have already shown in Fig. 4, but with several modifications with respect to that figure. Indeed, firstly we have depicted in a more simple and schematic way the subshrubs by showing one unique portion of each one of them; secondly, now we show

the chaotic bands that constitute the $\operatorname{shrub}_{0}\left(\frac{q_{1}}{p_{1}} \cdot \frac{q_{2}}{p_{2}} \cdots \frac{q_{N}}{p_{N}}\right)$ which were not shown in Fig. 4; and finally, now we start from the period-1 main cardioid $\frac{1}{1}$, i.e., we have also represented the generation route $\operatorname{gr}\left(\frac{q_{1}}{p_{1}} \cdot \frac{q_{2}}{p_{2}} \cdots \frac{q_{N}}{p_{N}}\right)$. 
As we said before in Section 3.2. (see Eq. 4) the $\operatorname{shrub}_{0}\left(\frac{q_{1}}{p_{1}} \cdot \frac{q_{2}}{p_{2}} \ldots \frac{q_{N}}{p_{N}}\right)$ is constituted by the infinite number of chaotic bands of the $N$-ary hyperbolic component $\frac{q_{1}}{p_{1}} \cdot \frac{q_{2}}{p_{2}} \cdots \frac{q_{N}}{p_{N}}$ : $\mathbf{B}_{0}\left(\frac{q_{1}}{p_{1}} \cdot \frac{q_{2}}{p_{2}} \cdots \frac{q_{N}}{p_{N}}\right), \mathbf{B}_{1}\left(\frac{q_{1}}{p_{1}} \cdot \frac{q_{2}}{p_{2}} \cdots \frac{q_{N}}{p_{N}}\right), \ldots \mathbf{B}_{\infty}\left(\frac{q_{1}}{p_{1}} \cdot \frac{q_{2}}{p_{2}} \cdots \frac{q_{N}}{p_{N}}\right)$. But, as we have shown in Pastor et al. [2005], each one of these chaotic bands can be obtained from a hyperbolic component of the period-doubling cascade: the chaotic band $\mathbf{B}_{0}$ from the hyperbolic component $\frac{q_{1}}{p_{1}} \cdot \frac{q_{2}}{p_{2}} \cdots \frac{q_{N}}{p_{N}}, \mathbf{B}_{1}$ from the first disc of the period-doubling cascade, $\mathbf{B}_{2}$ from the second one, and so on as far as both the hyperbolic components of the period-doubling cascade and the chaotic bands come together in the Myrberg-Feigenbaum point. That is to say, by using algorithms, the harmonics $H^{(i)}\left(\frac{q_{1}}{p_{1}} \cdots \frac{q_{N}}{p_{N}}\right), H^{(i)}\left(\frac{q_{1}}{p_{1}} \cdots \frac{q_{N}}{p_{N}} \cdot \frac{1}{2}\right), H^{(i)}\left(\frac{q_{1}}{p_{1}} \cdots \frac{q_{N}}{p_{N}} \cdot \frac{1}{2} \cdot \frac{1}{2}\right), \ldots$ [Pastor et al., 2005], we can calculate the last appearance hyperbolic components of the chaotic bands $\mathbf{B}_{0}\left(\frac{q_{1}}{p_{1}} \cdot \frac{q_{2}}{p_{2}} \cdots \frac{q_{N}}{p_{N}}\right)$, $\mathbf{B}_{1}\left(\frac{q_{1}}{p_{1}} \cdot \frac{q_{2}}{p_{2}} \cdots \frac{q_{N}}{p_{N}}\right) \quad \mathbf{B}_{2}\left(\frac{q_{1}}{p_{1}} \cdot \frac{q_{2}}{p_{2}} \cdots \frac{q_{N}}{p_{N}}\right), \ldots$ Therefore we say that $\left(\frac{q_{1}}{p_{1}} \cdots \frac{q_{N}}{p_{N}}\right)$ generates $\mathbf{B}_{0}$ (or that $\left(\frac{q_{1}}{p_{1}} \cdots \frac{q_{N}}{p_{N}}\right)$ is the gene of $\left.\mathbf{B}_{0}\right),\left(\frac{q_{1}}{p_{1}} \cdots \frac{q_{N}}{p_{N}} \cdot \frac{1}{2}\right)$ generates $\mathbf{B}_{1}$ (or that $\left(\frac{q_{1}}{p_{1}} \cdots \frac{q_{N}}{p_{N}} \cdot \frac{1}{2}\right)$ is the gene of $\mathbf{B}_{1}$ ), $\left(\frac{q_{1}}{p_{1}} \cdots \frac{q_{N}}{p_{N}} \cdot \frac{1}{2} \cdot \frac{1}{2}\right)$ generates $\mathbf{B}_{2}$ (or that $\left(\frac{q_{1}}{p_{1}} \cdots \frac{q_{N}}{p_{N}} \cdot \frac{1}{2} \cdot \frac{1}{2}\right)$ is the gene of $\mathbf{B}_{2}$ ), and so on.

We can clearly see it in Fig. 5, where the $\operatorname{shrub}_{0}\left(\frac{q_{1}}{p_{1}} \cdot \frac{q_{2}}{p_{2}} \cdots \frac{q_{N}}{p_{N}}\right)$ seems the specular image of the $\operatorname{MFr}\left(\frac{q_{1}}{p_{1}} \cdot \frac{q_{2}}{p_{2}} \cdots \frac{q_{N}}{p_{N}}\right)$ in a mirror placed at $\operatorname{MF}^{(N)}\left(\frac{q_{1}}{p_{1}} \cdot \frac{q_{2}}{p_{2}} \cdots \frac{q_{N}}{p_{N}}\right)$, as it happens in the onedimensional case. Now, when starting from the hyperbolic component $\left(\frac{q_{1}}{p_{1}} \cdot \frac{q_{2}}{p_{2}} \cdots \frac{q_{N}}{p_{N}}\right)$ we cover its period-doubling cascade towards the chaos we are determining how such a chaos will be in the $\operatorname{shrub}_{0}\left(\frac{q_{1}}{p_{1}} \cdot \frac{q_{2}}{p_{2}} \cdots \frac{q_{N}}{p_{N}}\right)$.

But, what is the matter with the remainder? We have not still seen here if there is any relation between the $\operatorname{shrub}_{\mathrm{r}}\left(\frac{q_{1}}{p_{1}} \cdot \frac{q_{2}}{p_{2}} \cdots \frac{q_{N}}{p_{N}}\right)$ and the generation route of the $\mathrm{N}$-ary hyperbolic component $\frac{q_{1}}{p_{1}} \cdot \frac{q_{2}}{p_{2}} \cdots \frac{q_{N}}{p_{N}}$. The shrub remainder is formed by $N$ subshrubs: $\operatorname{subshrub}_{1}\left(\frac{q_{1}}{p_{1}} \cdot \frac{q_{2}}{p_{2}} \cdots \frac{q_{N}}{p_{N}}\right), \operatorname{subshrub}_{2}\left(\frac{q_{1}}{p_{1}} \cdot \frac{q_{2}}{p_{2}} \cdots \frac{q_{N}}{p_{N}}\right) \ldots \quad \operatorname{subshrub}_{N}\left(\frac{q_{1}}{p_{1}} \cdot \frac{q_{2}}{p_{2}} \cdots \frac{q_{N}}{p_{N}}\right)$. The generation route to reach $\frac{q_{1}}{p_{1}} \cdot \frac{q_{2}}{p_{2}} \cdots \frac{q_{N}}{p_{N}}$ is also formed by $N$ hyperbolic components: $\frac{1}{1}, \frac{q_{1}}{p_{1}}, \frac{q_{1}}{p_{1}} \cdot \frac{q_{2}}{p_{2}}, \frac{q_{1}}{p_{1}} \cdot \frac{q_{2}}{p_{2}} \cdot \frac{q_{3}}{p_{3}}$, $\ldots, \frac{q_{1}}{p_{1}} \cdot \frac{q_{2}}{p_{2}} \ldots \frac{q_{N-2}}{p_{N-2}}$ and $\frac{q_{1}}{p_{1}} \cdot \frac{q_{2}}{p_{2}} \cdots \frac{q_{N-1}}{p_{N-1}}$. Are the $N$ subshrubs of the shrub ${ }_{\mathrm{r}}\left(\frac{q_{1}}{p_{1}} \cdot \frac{q_{2}}{p_{2}} \cdots \frac{q_{N}}{p_{N}}\right)$ related with the $N$ hyperbolic components of the $\operatorname{gr}\left(\frac{q_{1}}{p_{1}} \cdot \frac{q_{2}}{p_{2}} \cdots \frac{q_{N}}{p_{N}}\right)$ ? Or more specifically, are the $N$ hyperbolic components of the generation route the genes of the $N$ subshrubs of the shrub remainder? Let us now see that this is so. 
We have just seen that the hyperbolic components of the MF route were the genes of $\mathbf{B}_{0}\left(\frac{q_{1}}{p_{1}} \cdot \frac{q_{2}}{p_{2}} \cdots \frac{q_{N}}{p_{N}}\right), \mathbf{B}_{1}\left(\frac{q_{1}}{p_{1}} \cdot \frac{q_{2}}{p_{2}} \cdots \frac{q_{N}}{p_{N}}\right), \ldots \mathbf{B}_{\infty}\left(\frac{q_{1}}{p_{1}} \cdot \frac{q_{2}}{p_{2}} \cdots \frac{q_{N}}{p_{N}}\right)$ because we have an algorithm to calculate the last appearance hyperbolic components of these chaotic bands starting from these genes [Pastor et al., 2005]. Unfortunately we have not as yet an algorithm to calculate the last appearance hyperbolic components of the subshrubs starting from the hyperbolic components of the generation route. However, we also saw that the characteristic Misiurewicz points of a chaotic band indicate the gene of such a chaotic band [Pastor et al., 2004]. Therefore, we can use the characteristic Misiurewicz points of a subshrub, that are the more visible nodes of the subshrub, to find the gene of such a subshrub. As we know from Romera et al. [2003] and Pastor et al. [2004], the characteristic Misiurewicz points $M_{n^{*}, p^{*}}$ (sometimes named by typing error non-characteristic Misiurewicz points in Romera et al. [2003]) of the $\operatorname{subshrub}_{1}\left(\frac{q_{1}}{p_{1}} \cdot \frac{q_{2}}{p_{2}} \cdots \frac{q_{N}}{p_{N}}\right), \quad \operatorname{subshrub}_{2}\left(\frac{q_{1}}{p_{1}} \cdot \frac{q_{2}}{p_{2}} \cdots \frac{q_{N}}{p_{N}}\right) \quad \ldots \quad \operatorname{subshrub}_{N-1}\left(\frac{q_{1}}{p_{1}} \cdot \frac{q_{2}}{p_{2}} \cdots \frac{q_{N}}{p_{N}}\right) \quad$ and $\operatorname{subshrub}_{N}\left(\frac{q_{1}}{p_{1}} \cdot \frac{q_{2}}{p_{2}} \cdots \frac{q_{N}}{p_{N}}\right)$, have periods $p^{*}=p_{1} \cdot p_{2} \cdots p_{N-1}, p^{*}=p_{1} \cdot p_{2} \cdots p_{N-2}, \ldots, p^{*}=p_{1}$ and $p^{*}=1$. Therefore, subshrub $1\left(\frac{q_{1}}{p_{1}} \cdot \frac{q_{2}}{p_{2}} \cdots \frac{q_{N}}{p_{N}}\right)$ come from $\frac{q_{1}}{p_{1}} \cdot \frac{q_{2}}{p_{2}} \cdots \frac{q_{N-1}}{p_{N-1}}$ (or $\frac{q_{1}}{p_{1}} \cdot \frac{q_{2}}{p_{2}} \cdots \frac{q_{N-1}}{p_{N-1}}$ is the gene of $\operatorname{subshrub}_{1}\left(\frac{q_{1}}{p_{1}} \cdot \frac{q_{2}}{p_{2}} \cdots \frac{q_{N}}{p_{N}}\right)$ ); $\operatorname{subshrub}_{2}\left(\frac{q_{1}}{p_{1}} \cdot \frac{q_{2}}{p_{2}} \cdots \frac{q_{N}}{p_{N}}\right)$ come from $\frac{q_{1}}{p_{1}} \cdot \frac{q_{2}}{p_{2}} \cdots \frac{q_{N-2}}{p_{N-2}}$ (or $\frac{q_{1}}{p_{1}} \cdot \frac{q_{2}}{p_{2}} \cdots \frac{q_{N-2}}{p_{N-2}}$ is the gene of $\left.\operatorname{subshrub}_{2}\left(\frac{q_{1}}{p_{1}} \cdot \frac{q_{2}}{p_{2}} \cdots \frac{q_{N}}{p_{N}}\right)\right) ; \ldots ; \operatorname{subshrub}_{N-2}\left(\frac{q_{1}}{p_{1}} \cdot \frac{q_{2}}{p_{2}} \cdots \frac{q_{N}}{p_{N}}\right)$ come from $\frac{q_{1}}{p_{1}} \cdot \frac{q_{2}}{p_{2}}$ (or $\frac{q_{1}}{p_{1}} \cdot \frac{q_{2}}{p_{2}}$ is the gene of $\left.\operatorname{subshrub}_{N-2}\left(\frac{q_{1}}{p_{1}} \cdot \frac{q_{2}}{p_{2}} \cdots \frac{q_{N}}{p_{N}}\right)\right)$; $\operatorname{subshrub}_{N-1}\left(\frac{q_{1}}{p_{1}} \cdot \frac{q_{2}}{p_{2}} \cdots \frac{q_{N}}{p_{N}}\right)$ come from $\frac{q_{1}}{p_{1}}$ (or $\frac{q_{1}}{p_{1}}$ is the gene of $\left.\operatorname{subshrub}_{N-1}\left(\frac{q_{1}}{p_{1}} \cdot \frac{q_{2}}{p_{2}} \cdots \frac{q_{N}}{p_{N}}\right)\right)$; and $\operatorname{subshrub}_{N}\left(\frac{q_{1}}{p_{1}} \cdot \frac{q_{2}}{p_{2}} \cdots \frac{q_{N}}{p_{N}}\right)$ come from the main cardioid $\frac{1}{1}$ ( or $\frac{1}{1}$ is the gene of $\operatorname{subshrub}_{N}\left(\frac{q_{1}}{p_{1}} \cdot \frac{q_{2}}{p_{2}} \cdots \frac{q_{N}}{p_{N}}\right)$ ).

By generalizing, as we know from Romera et al. [2003], if the node $M_{n^{*}, p^{*}}$ is a characteristic Misiurewicz point of a $\operatorname{subshrub}_{i}\left(\frac{q_{1}}{p_{1}} \cdot \frac{q_{2}}{p_{2}} \cdots \frac{q_{N}}{p_{N}}\right), 1 \leq i \leq N$, then it has a period $p_{i}^{*}=p_{1} \cdot p_{2} \cdots p_{N-i}$ (when $i=N$ then $p_{i}^{*}=p_{0}=1$ ). Therefore, $\frac{q_{1}}{p_{1}} \cdot \frac{q_{2}}{p_{2}} \cdots \frac{q_{N-i}}{p_{N-i}}$ is the gene of $\operatorname{subshrub}_{i}\left(\frac{q_{1}}{p_{1}} \cdot \frac{q_{2}}{p_{2}} \cdots \frac{q_{N}}{p_{N}}\right), 1 \leq i \leq N$ (when $i=N$ the gene is $\left.\frac{q_{0}}{p_{0}}=\frac{1}{1}\right)$.

In Fig. 5 we have depicted clearly the correspondence between subshrub $\left(\frac{q_{1}}{p_{1}} \cdot \frac{q_{2}}{p_{2}} \cdots \frac{q_{N}}{p_{N}}\right)$ and $\operatorname{gr}\left(\frac{q_{1}}{p_{1}} \cdot \frac{q_{2}}{p_{2}} \cdots \frac{q_{N}}{p_{N}}\right)$. Also here $\operatorname{shrub}_{\mathrm{r}}\left(\frac{q_{1}}{p_{1}} \cdot \frac{q_{2}}{p_{2}} \cdots \frac{q_{N}}{p_{N}}\right)$ seems the specular image of $\operatorname{gr}\left(\frac{q_{1}}{p_{1}} \cdot \frac{q_{2}}{p_{2}} \cdots \frac{q_{N}}{p_{N}}\right)$ 
in a mirror placed at $\operatorname{MF}^{(N)}\left(\frac{q_{1}}{p_{1}} \cdot \frac{q_{2}}{p_{2}} \cdots \frac{q_{N}}{p_{N}}\right)$. That is to say, $\operatorname{gr}\left(\frac{q_{1}}{p_{1}} \cdot \frac{q_{2}}{p_{2}} \cdots \frac{q_{N}}{p_{N}}\right)$ is also determining the chaos in $\operatorname{shrub}_{\mathrm{r}}\left(\frac{q_{1}}{p_{1}} \cdot \frac{q_{2}}{p_{2}} \cdots \frac{q_{N}}{p_{N}}\right)$.

As we have just seen, the gene of the $\operatorname{subshrub}_{i}\left(\frac{q_{1}}{p_{1}} \cdot \frac{q_{2}}{p_{2}} \cdots \frac{q_{N}}{p_{N}}\right)$ is $\frac{q_{1}}{p_{1}} \cdot \frac{q_{2}}{p_{2}} \cdots \frac{q_{N-i}}{p_{N-i}}$, with period $p_{i}^{*}=p_{1} \cdot p_{2} \cdots p_{N-i}$, whose last factor is $p_{N-i}$. This last value has not to be mistaken with the number of branches emanating from a node in the $\operatorname{subshrub}_{i}\left(\frac{q_{1}}{p_{1}} \cdot \frac{q_{2}}{p_{2}} \cdots \frac{q_{N}}{p_{N}}\right)$, that is $p_{N-i+1}$, $1 \leq i \leq N$, as we saw in Section 3.2. Thus, for $i=N$ the gene of $\operatorname{subshrub}_{N}\left(\frac{q_{1}}{p_{1}} \cdot \frac{q_{2}}{p_{2}} \cdots \frac{q_{N}}{p_{N}}\right)$ is $\frac{1}{1}$, but the number of branches emanating from each node of $\operatorname{subshrub}_{N}\left(\frac{q_{1}}{p_{1}} \cdot \frac{q_{2}}{p_{2}} \cdots \frac{q_{N}}{p_{N}}\right)$ is $p_{1}$; or for $i=1$ the gene of $\operatorname{subshrub}_{1}\left(\frac{q_{1}}{p_{1}} \cdot \frac{q_{2}}{p_{2}} \cdots \frac{q_{N}}{p_{N}}\right)$ is $\frac{q_{1}}{p_{1}} \cdot \frac{q_{2}}{p_{2}} \cdots \frac{q_{N-1}}{p_{N-1}}$, but the number of branches emanating from each node of subshrub $1\left(\frac{q_{1}}{p_{1}} \cdot \frac{q_{2}}{p_{2}} \cdots \frac{q_{N}}{p_{N}}\right)$ is $p_{N}$.

But we have still more. We have treated the relation between the chaotic region and the periodic region in two separated parts: $\operatorname{MFr}\left(\frac{q_{1}}{p_{1}} \cdot \frac{q_{2}}{p_{2}} \cdots \frac{q_{N}}{p_{N}}\right)$ is related with $\operatorname{shrub}_{0}\left(\frac{q_{1}}{p_{1}} \cdot \frac{q_{2}}{p_{2}} \cdots \frac{q_{N}}{p_{N}}\right)$, and $\operatorname{gr}\left(\frac{q_{1}}{p_{1}} \cdot \frac{q_{2}}{p_{2}} \cdots \frac{q_{N}}{p_{N}}\right)$ is related with $\operatorname{shrub}_{\mathrm{r}}\left(\frac{q_{1}}{p_{1}} \cdot \frac{q_{2}}{p_{2}} \cdots \frac{q_{N}}{p_{N}}\right)$. However, we shall see that we can use one an? unique relation between the whole periodic region and the whole chaotic region.

As we can clearly see in Fig. 5, on the one hand we denominate periodic route through $\frac{q_{1}}{p_{1}} \cdot \frac{q_{2}}{p_{2}} \cdots \frac{q_{N}}{p_{N}}, \operatorname{pr}\left(\frac{q_{1}}{p_{1}} \cdot \frac{q_{2}}{p_{2}} \cdots \frac{q_{N}}{p_{N}}\right)$, to the generation route of the hyperbolic component $\frac{q_{1}}{p_{1}} \cdot \frac{q_{2}}{p_{2}} \cdots \frac{q_{N}}{p_{N}}$ followed by the Myrberg-Feigenbaum route of the hyperbolic component $\frac{q_{1}}{p_{1}} \cdot \frac{q_{2}}{p_{2}} \cdots \frac{q_{N}}{p_{N}}$. Therefore,

$$
\operatorname{pr}\left(\frac{q_{1}}{p_{1}} \cdot \frac{q_{2}}{p_{2}} \cdots \frac{q_{N}}{p_{N}}\right)=\operatorname{gr}\left(\frac{q_{1}}{p_{1}} \cdot \frac{q_{2}}{p_{2}} \cdots \frac{q_{N}}{p_{N}}\right) \cup \operatorname{MFr}\left(\frac{q_{1}}{p_{1}} \cdot \frac{q_{2}}{p_{2}} \cdots \frac{q_{N}}{p_{N}}\right)
$$

On the other hand, as we already saw in Eq. (3), $\operatorname{shrub}\left(\frac{q_{1}}{p_{1}} \cdot \frac{q_{2}}{p_{2}} \cdots \frac{q_{N}}{p_{N}}\right)$ is constituted by $\operatorname{shrub}_{0}\left(\frac{q_{1}}{p_{1}} \cdot \frac{q_{2}}{p_{2}} \ldots \frac{q_{N}}{p_{N}}\right)$ followed by $\operatorname{shrub}_{\mathrm{r}}\left(\frac{q_{1}}{p_{1}} \cdot \frac{q_{2}}{p_{2}} \cdots \frac{q_{N}}{p_{N}}\right)$. As we shall see now, we can treat on the one hand all the periodic routes and on the other all the shrubs (chaotic routes).

Indeed, the separation between $\operatorname{shrub}_{0}\left(\frac{q_{1}}{p_{1}} \cdot \frac{q_{2}}{p_{2}} \cdots \frac{q_{N}}{p_{N}}\right)$ and $\operatorname{shrub}_{\mathrm{r}}\left(\frac{q_{1}}{p_{1}} \cdot \frac{q_{2}}{p_{2}} \cdots \frac{q_{N}}{p_{N}}\right)$, even though sometimes very useful, is artificial since both have the same nature. Certainly, as we know from Pastor et al. [2005], shrub $0\left(\frac{q_{1}}{p_{1}} \cdot \frac{q_{2}}{p_{2}} \cdots \frac{q_{N}}{p_{N}}\right)$ has an infinity of subshrubs or chaotic bands 
that can be obtained from the period-doubling cascade of $\frac{q_{1}}{p_{1}} \cdot \frac{q_{2}}{p_{2}} \cdots \frac{q_{N}}{p_{N}}: \frac{q_{1}}{p_{1}} \cdot \frac{q_{2}}{p_{2}} \cdots \frac{q_{N}}{p_{N}}$, $\frac{q_{1}}{p_{1}} \cdot \frac{q_{2}}{p_{2}} \cdots \frac{q_{N}}{p_{N}} \cdot \frac{1}{2}, \frac{q_{1}}{p_{1}} \cdot \frac{q_{2}}{p_{2}} \cdots \frac{q_{N}}{p_{N}} \cdot \frac{1}{2} \cdot \frac{1}{2} \ldots$. Since the last fraction of each one of the hyperbolic components (starting from the second one) is $\frac{1}{2}$, the infinity of the above subshrubs have two branches emanating from each node and therefore $\operatorname{shrub}_{0}\left(\frac{q_{1}}{p_{1}} \cdot \frac{q_{2}}{p_{2}} \cdots \frac{q_{N}}{p_{N}}\right)$ seems a single branch. Summarizing, both $\operatorname{shrub}_{\mathrm{r}}\left(\frac{q_{1}}{p_{1}} \cdot \frac{q_{2}}{p_{2}} \cdots \frac{q_{N}}{p_{N}}\right)$ and $\operatorname{shrub}_{0}\left(\frac{q_{1}}{p_{1}} \cdot \frac{q_{2}}{p_{2}} \ldots \frac{q_{N}}{p_{N}}\right)$ are constituted by subshrubs, the former by $\mathrm{N}$ subshrubs and the latter by an infinity of subshrubs.

Likewise, in a periodic route, the generation route and the Myrberg-Feigenbaum route have also the same nature because both are constituted by hyperbolic components of the periodic region, all of them discs, except for the main cardioid. The generation route have $\mathrm{N}$ hyperbolic components and the Myrgerg-Feigenbaum route an infinity of hyperbolic components.

Obviously, a periodic route is related with its chaotic route since the two parts of them are related. As we can see in Fig. 5, $\operatorname{pr}\left(\frac{q_{1}}{p_{1}} \cdot \frac{q_{2}}{p_{2}} \cdots \frac{q_{N}}{p_{N}}\right)$ seems the specular image of $\operatorname{shrub}\left(\frac{q_{1}}{p_{1}} \cdot \frac{q_{2}}{p_{2}} \cdots \frac{q_{N}}{p_{N}}\right)$ in a mirror placed at $\operatorname{MF}^{(N)}\left(\frac{q_{1}}{p_{1}} \cdot \frac{q_{2}}{p_{2}} \cdots \frac{q_{N}}{p_{N}}\right)$. That is to say, the periodic route to reach the chaos through the hyperbolic component $\frac{q_{1}}{p_{1}} \cdot \frac{q_{2}}{p_{2}} \ldots \frac{q_{N}}{p_{N}}$ determines how the chaos of the total $\operatorname{shrub}\left(\frac{q_{1}}{p_{1}} \cdot \frac{q_{2}}{p_{2}} \cdots \frac{q_{N}}{p_{N}}\right)$ will be, what it is a generalization of the one-dimensional case. In other words, in the same way as in the 1D quadratic maps the structure of chaotic bands copies the structure of the period-doubling cascade, in the Mandelbrot set the structure of the shrubs (chaotic routes) copies the structure of the corresponding periodic route. What we have just seen occurs in any hyperbolic component $\frac{q_{1}}{p_{1}} \cdot \frac{q_{2}}{p_{2}} \cdots \frac{q_{N}}{p_{N}}$. Each one of these hyperbolic components provides one periodic route through itself; therefore, the former generalization takes place as times as hyperbolic components $\frac{q_{1}}{p_{1}} \cdot \frac{q_{2}}{p_{2}} \ldots \frac{q_{N}}{p_{N}}$ there are in the periodic region.

Hence, the periodic region is like a general index of what the chaotic region will be. If we choose a particular case of this general index, one specific periodic route towards the chaos, when we cross the corresponding Myrberg-Feigenbaum point we reach a particular zone of the chaotic region whose shrub is determined by the hyperbolic components of such a periodic route. 


\section{Conclusions}

We begin by remembering the close relation between the period-doubling cascade hyperbolic components of the periodic region and the chaotic bands of the chaotic region in 1D quadratic maps. Next, we have posed the possible relation between the periodic and chaotic regions of the Mandelbrot set. In order to see it, we analyse separately the periodic and chaotic regions of this set. The role plaid by the period-doubling cascade in 1D quadratic maps, in the case of the periodic region of the Mandelbrot set is plaid by each one of the infinite number of periodic routes. Likewise, the role plaid by the chaotic bands in 1D quadratic maps, in the case of the chaotic region of the Mandelbrot set is plaid by the shrubs. Thus, the periodic route through a given hyperbolic component is fixing how will be the shrub of such a hyperbolic component. Therefore, the periodic region of the Mandelbrot set is related to its chaotic region since the first one determines how will be the second one.

\section{Acknowledgments}

This work was supported by "Ministerio de Educación y Ciencia" of Spain, research grant SEG2004-02418. 


\section{References}

Branner, B. [1989] "The Mandelbrot set," in Chaos and Fractals: The Mathematics Behind the Computer Graphics, eds. Devaney, R. L. \& Keen, L. (AMS, Providence), pp. 75-105.

Devaney, R. L. [1989] An Introduction to Chaotic Dynamical Systems (Addison-Wesley, NY) pp. 44-48.

Devaney, R. L. [1995] "The fractal geometry of the Mandelbrot set. How to count and how to add," Fractals 3, 629-640.

Douady, A. \& Hubbard, J. H. [1985] "Etude dynamique des polynômes complexes," Publ. Math. d'Orsay, 85-04., pp. 56-60.

Eckmann, J.-P. [1981] "Roads to turbulence in dissipative dynamical systems," Rev. Mod. Phys. 53, 643-654.

Grossmann, S. \& Thomae, Z. [1977] "Invariant distributions and stationary correlation functions of one-dimensional discrete processes," Z. Naturforsch A32, 1353-1363.

Hale, J. \& Koçak, H. [1991] Dynamics and Bifurcations (Springer-Verlag, NY).

Hao, B.-L. \& Zheng, W.-M. [1998] Applied Symbolic Dynamics and Chaos (World Scientific, Singapore) pp. 13-100.

Lauterborn, W. \& Parlitz, U. [1988] "Methods of chaos physics and their applications to acoustics," J. Acoust. Soc. Am. 84, 1975-1993.

Lorenz, E.N. [1980] "Noise periodicity and reverse bifurcation," Annals of the New York Academy of Sciences 357, 282-291.

Mandelbrot, B. B. [1980] "Fractal aspects of the iteration of $z \rightarrow \lambda z(1-z)$ for complex $\lambda$ and $z$," in Nonlinear Dynamics, ed. Helleman, R. H. G. (Annals of the New York Academy of Sciences, NY), pp. 249-259.

Mandelbrot, B. B. [1983] "On the quadratic mapping $z \rightarrow z^{2}-\mu$ for complex $\mu$ and $z$ : the fractal structure of its $M$ set, and scaling," Physica D7, 224-239.

Milnor, J. \& Thurston, W. [1988] “On iterated maps of the interval," in Dynamical Systems, ed. Alexander, J. C. (Springer, Berlin) pp. 465-563.

Milnor, J. [1989] "Self-similarity and hairiness in the Mandelbrot set," in Computers in Geometry and Topology, ed. Tangora, Lecture Notes in Pure and Applied Mathematics, Vol. 114 (Dekker), pp. 211-257.

Misiurewicz, M. \& Nitecki, Z. [1991] "Combinatorial patterns for maps of the interval," Mem. Am. Math. Soc. 94, 1-110. 
Pastor, G., Romera, M. \& Montoya, F. [1996] "An approach to the ordering of onedimensional quadratic maps," Chaos, Solitons and Fractals 7, 565-584.

Pastor, G., Romera, M. \& Montoya, F. [1997] "Harmonic structure of one-dimensional quadratic maps," Phys. Rev. E56, 1476-1483.

Pastor, G., Romera, M., Alvarez, G. \& Montoya, F. [2001] "Misiurewicz point patterns generation in one-dimensional quadratic maps," Physica A292, 207-230.

Pastor, G., Romera M., Alvarez, G. \& Montoya, F. [2002] "Operating with external arguments in the Mandelbrot set antenna," Physica D171, 52-71.

Pastor, G., Romera, M., Alvarez, G. \& Montoya, F. [2004] "Chaotic bands in the Mandelbrot set," Comput. \& Graph. 28, 779-784.

Pastor, G., Romera, M., Alvarez, G. \& Montoya, F. [2005] "External arguments for the chaotic bands calculation in the Mandelbrot set," Physica A353 145-158.

Peitgen, H. O. \& Richter, P. H. [1986] The Beauty of Fractals (Springer-Verlag, Berlin).

Zheng, W.-M. \& Hao, B.-L. [1990] “Applied symbolic dynamics," in Experimental Study and Characterization of Chaos, ed. Hao, B.-L. (World Scientific, Singapore) pp. 364-459.

Romera, M., Pastor, G. \& Montoya, F. [1996] "Misiurewicz points in one-dimensional quadratic maps," Physica A232, 517-535.

Romera, M., Pastor, G., Alvarez, G. \& Montoya, F. [2003] "Shrubs in the Mandelbrot set ordering," Int. J. Bifurcat. Chaos 13, 2279-2300.

Shaw, R. [1981] "Strange attractors, chaotic behavior, and information flow," Z. Naturforsch A36, 80-112. 


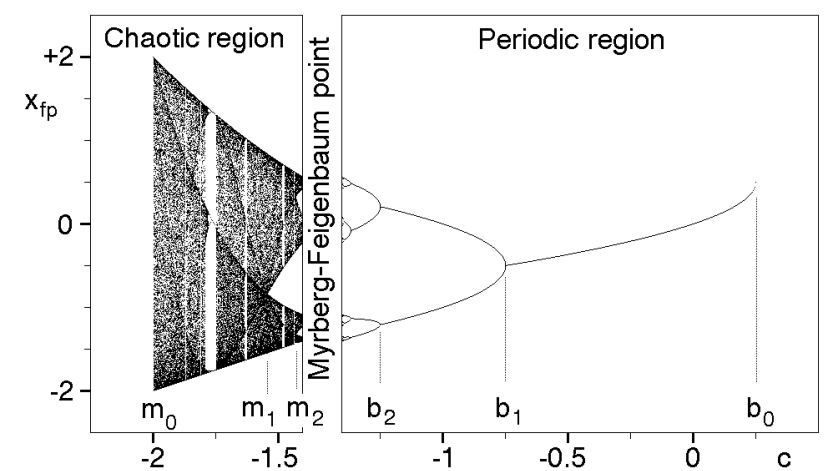

(a)

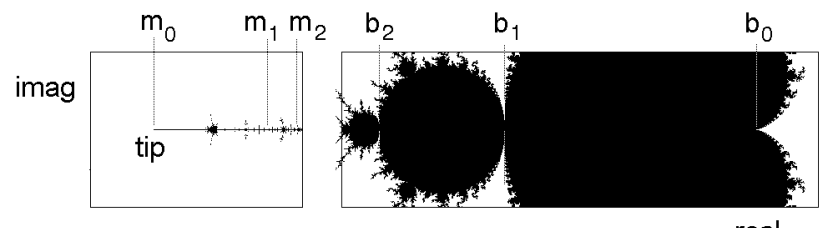

(b)

Fig. 1. (a) Bifurcation diagram of the Mandelbrot real map $x_{n+1}=x_{n}^{2}+c$. (b) The neighborhood of the real part of the Mandelbrot set $z_{n+1}=z_{n}^{2}+c$. 


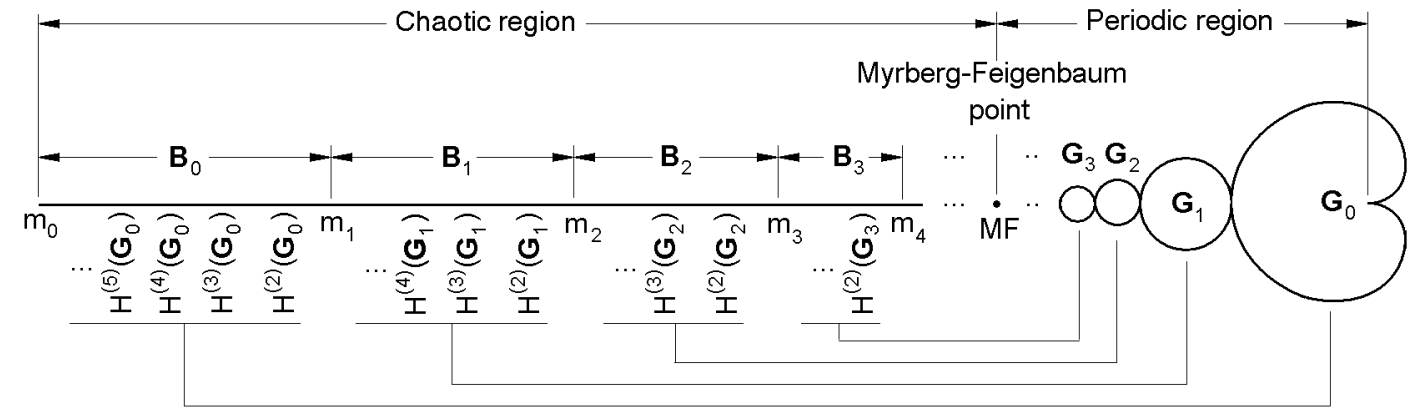

Fig. 2. A sketch of the Mandelbrot real map $x_{n+1}=x_{n}^{2}+c$ by using the neighborhood of the real part of the Mandelbrot set. 


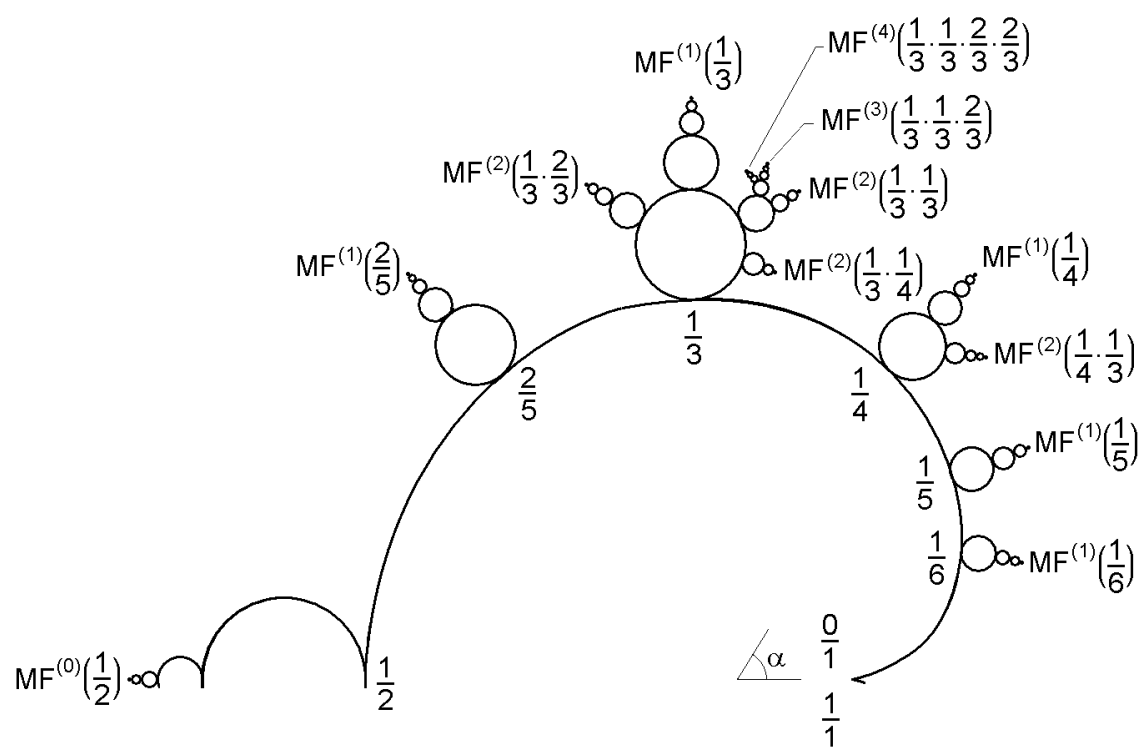

Fig. 3. A sketch of the periodic region of the Mandelbrot set. 


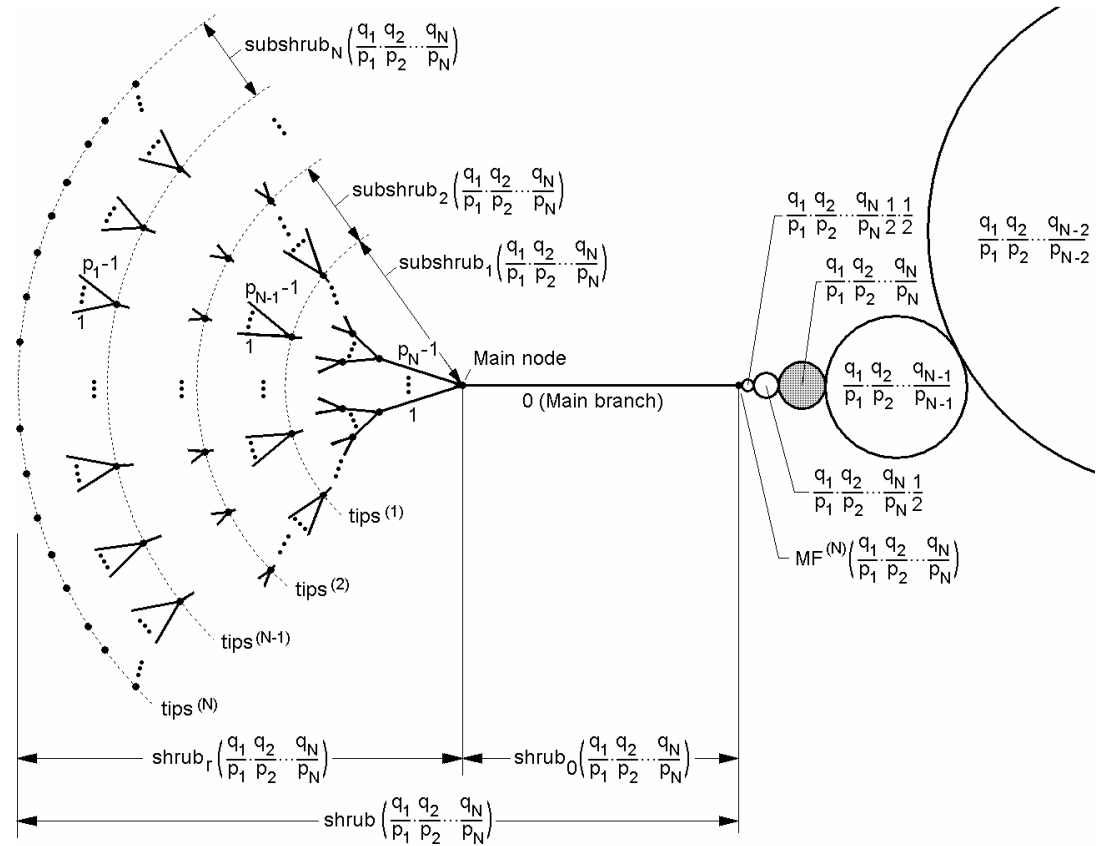

Fig. 4. A sketch of the shrub of a $N$-ary hyperbolic component, $\operatorname{shrub}\left(\frac{q_{1}}{p_{1}} \cdot \frac{q_{2}}{p_{2}} \cdots \frac{q_{N}}{p_{N}}\right)$, where both the $\operatorname{shrub}_{0}\left(\frac{q_{1}}{p_{1}} \cdot \frac{q_{2}}{p_{2}} \cdots \frac{q_{N}}{p_{N}}\right)$ and the $N$ subshrubs of the $\operatorname{shrub}_{\mathrm{r}}\left(\frac{q_{1}}{p_{1}} \cdot \frac{q_{2}}{p_{2}} \cdots \frac{q_{N}}{p_{N}}\right)$ are shown. 


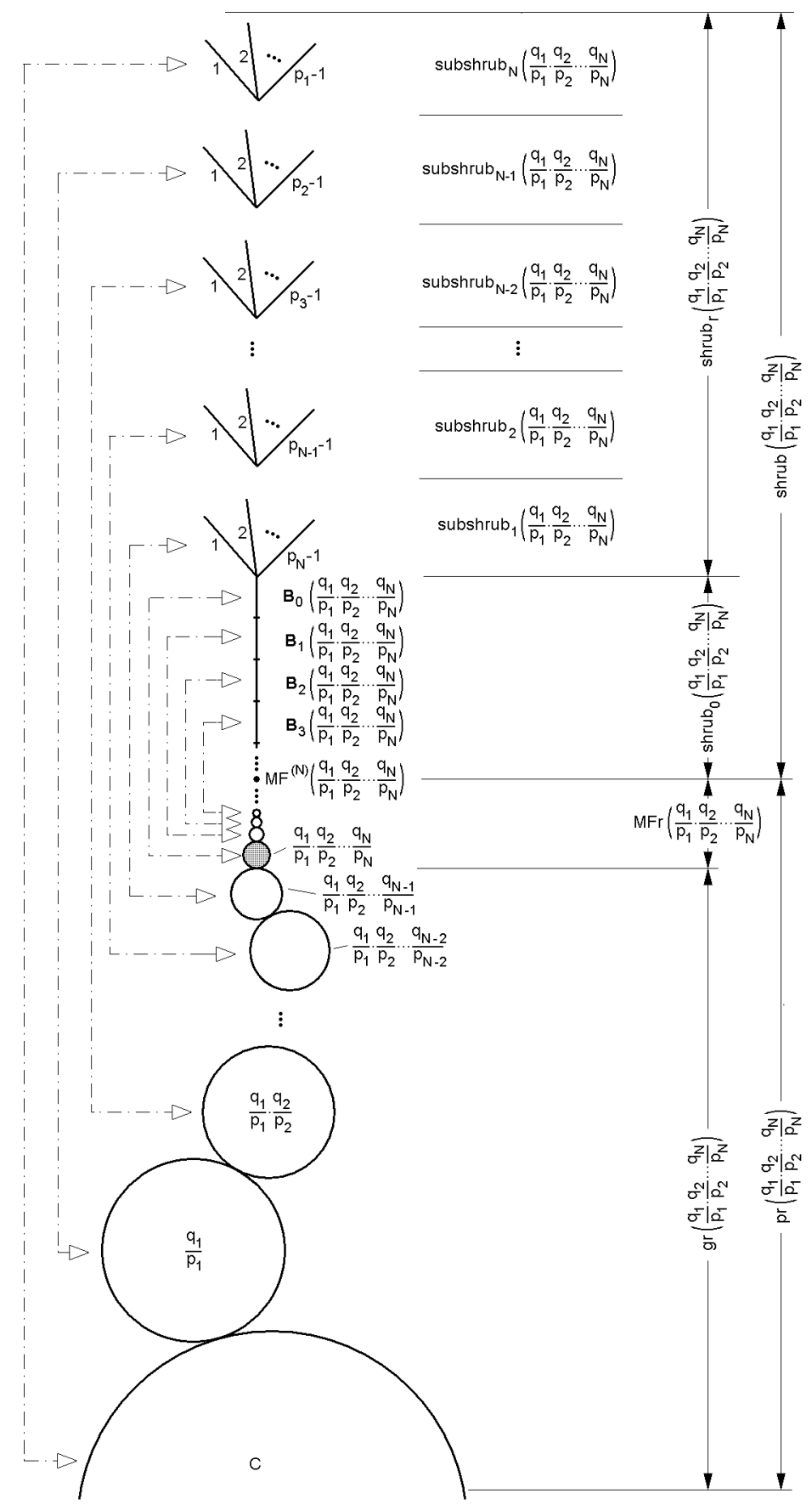

Fig. 5. Another sketch of the $\operatorname{shrub}\left(\frac{q_{1}}{p_{1}} \cdot \frac{q_{2}}{p_{2}} \cdots \frac{q_{N}}{p_{N}}\right)$ of a $N$-ary hyperbolic component shown in Fig. 4. Here we show explicitly $\operatorname{shrub}_{0}\left(\frac{q_{1}}{p_{1}} \cdot \frac{q_{2}}{p_{2}} \cdots \frac{q_{N}}{p_{N}}\right)$, we show more schematically $\operatorname{shrub}_{\mathrm{r}}\left(\frac{q_{1}}{p_{1}} \cdot \frac{q_{2}}{p_{2}} \cdots \frac{q_{N}}{p_{N}}\right)$ and we give all the periodic route to chaos throw $\frac{q_{1}}{p_{1}} \cdot \frac{q_{2}}{p_{2}} \cdots \frac{q_{N}}{p_{N}}$, $\operatorname{pr}\left(\frac{q_{1}}{p_{1}} \cdot \frac{q_{2}}{p_{2}} \cdots \frac{q_{N}}{p_{N}}\right)$. 